\title{
A semi-stochastic cell-based formalism to model the dynamics of migration of cells in colonies
}

\author{
F. J. Vermolen - A. Gefen
}

Received: 1 November 2010 / Accepted: 12 March 2011 / Published online: 26 March 2011

(C) The Author(s) 2011. This article is published with open access at Springerlink.com

\begin{abstract}
We consider the movement and viability of individual cells in cell colonies. Cell movement is assumed to take place as a result of sensing the strain energy density as a mechanical stimulus. The model is based on tracking the displacement and viability of each individual cell in a cell colony. Several applications are shown, such as the dynamics of filling a gap within a fibroblast colony and the invasion of a cell colony. Though based on simple principles, the model is qualitatively validated by experiments on living fibroblasts on a flat substrate.
\end{abstract}

Keywords Cell migration · Cell-based model ·

Semi-stochastic model

\section{Introduction}

Processes like wound healing and tumor growth involve biological processes like cell migration, cell proliferation, cell differentiation, and apoptosis (programmed cell death). Possible mechanisms for cell migration include random walk, biased random walk, positive or negative chemotaxis (cell motion toward or opposite the gradient of a chemical like a growth factor) or mechanotaxis (cell mobility as a result of a mechanical stimulus). Since experiments are sometimes hard to carry out, or time consuming, or expensive, or hard from an ethical point of view, computational methods can be

\section{F. J. Vermolen $(\varangle)$}

Delft Institute of Applied Mathematics,

Delft University of Technology, Delft, The Netherlands

e-mail: F.J.Vermolen@tudelft.nl

\section{A. Gefen}

Department of Biomedical Engineering, Tel Aviv University, 69978 Tel Aviv, Israel used to provide an easy and quick access to insights that one could hardly obtain otherwise. In the literature, many mathematical models describing processes like wound healing and tumor growth based on solving partial differential equations for several cell types and growth factors can be found. Most of the modeling studies on wound healing consider only one partial process. The papers on continuum hypothesis-based models for wound healing can be classified into

- models for contraction, for instance the work by Olsen et al. (1995), Murray (2004), Vermolen (2009), where both mechanistic effects from pulling forces exerted by (myo-)fibroblasts, proliferation, cell mobility and chemical interactions are dealt with;

- models for wound angiogenesis, in which we mention, among many others, the studies carried out by Maggelakis (2004), Schugart et al. (2008), Xue et al. (2009), and Gaffney (2002). In some of these studies, the shortage of oxygen is taken into account as a trigger for angiogenesis. Further, a distinction between capillaries (endothelial cells) and capillary tips is made in some of these studies. The partial differential equations have a deterministic nature, though their derivation is based on probabilistic principles, see Gaffney (2002);

- models for epidermal closure, in which keratinocyte migration and proliferation are responsible for the process of closure. Here, we mention the studies from Sherratt et al. (1991) and Vermolen and Javierre (2009). The first model describes a traveling wave analysis, whereas the second paper assesses fundamental mathematical questions on existence, uniqueness of solutions, and the mathematical nature of the moving boundary separating the wound from the undamaged tissue. Javierre et al. (2009) presents a numerical solution method for the moving boundary problem. 
Next to the use of partial differential equations, the cellular Potts model, or referred to as the Glazier-Graner-Hogeweg model, developed by Graner and Glazier (1992) is used to simulate biological processes, such as vascularization around tumors. Vascularization has been modeled using the cellular potts model extensively by Merks and Koolwijk (2009), among others. The cellular potts model is a lattice-based model in which each pixel can represent a cell and hence falls within the class of discrete cellular automata models. In the cellular potts model, the driving force of the movement of the cells is a Hamiltonian, that is an energy, which determines the probability of allowing a lattice change in terms of the positions of the entities (in most biological cases individual cells). Other applications of these discrete models were developed and described in studies by Plank and Sleeman (2004) and Dallon and Ehrlich (2008), Dallon (2010).

It has been suggested that the cell-substrate adhesion sites act like local mechanosensors and convert mechanical forces into biochemical signaling Schwarz and Bischofs (2005). There is empirical evidence that this signaling process is influenced by the stiffness of the substrate and it has been further established that fast cell migration occurs at intermediate substrate stiffnesses, whereas on stiff (or on extremely soft) substrata, slower migration takes place Sarvestani (2010). Reinhart-King et al. (2008) were the first investigators to evidence communication of cells through a substrate. In our study, we propose an alternative approach to all the aforementioned models, which takes the mechanism of cell sensing via the substrate into account, and with several characteristics in common with the Potts model, based on tracking each individual cell in a colony. The cells are allowed to move as a result of their sensing the strain energy density from their nearest and even far away neighbors, and, opposed to the cellular automata models, their positions are not constrained to lattice points in the current model. This type of modeling resembles the particle models (or discrete element models) that are commonly employed in the physical sciences, where particles in a flowing environment are tracked, see for instance Luding (2008). We realize that for large cell colonies, these computations get very elaborate, and that an optimization of the algorithms is crucial. In this paper, we compare numerical simulations to a simple analytic solution and we compare the quantitative behavior of the cell movements predicted by the current model to cell movements in an experimental setting. Since there is a huge number of wound healing models that are based on (systems of) partial differential equations, one of our future aims is a comparison of the partial differential equations based models to the current cell-based formalism. This comparison to simulations obtained from partial differential equations is not discussed in the present manuscript.

As first input, the initial positions of all cells need to be fed into the model. Each viable cell on the substrate exerts an upward traction force on the substrate. By this traction force, the substrate is distorted around the cell. This distortion is felt by the other cells, which distort the substrate as well by acting their upward pulling force. The distortion field, expressed by the strain energy density, that is sensed by a cell, makes the cell move in a particular direction. This holds for all cells, hence the distribution of the cells at the locations alters. Further, depending on the condition of the cells, such as age and viability, and on the environment of the substrate, such as toxicity and $\mathrm{Ph}$, the cells exert a specific traction on the substrate and move at a specific speed. In this paper, we track the motion of the cells, in which also the cell viability and cell division are taken into account. The current formalism is based on very simple, though elegant principles, such that the number of parameters is reduced by introducing phenomelogical relations rather than keeping complicated mechanistic formulations.

In Sect. 2, we introduce the mathematical formalism and the algorithm to solve the problem. This is followed by an analytic solution for a two-cell system in Sect. 3, where the numerical calculations are validated using the analytic solution. In Sect. 4, we present several case studies with two cells and with larger cell colonies. This is followed by a discussion on the strength and limitations of the model in Sect. 5 and a qualitative comparison with cell culture experiments. Finally, we give some conclusions in Sect. 6.

\section{The mathematical framework}

We are aware of the complexity of the geometry of cells, in particular when they are pulling and moving. As in a neutral (non-active) state, the projection of the cells onto the two-dimensional substrate resembles a circle. Therefore, we assume the cells to be hemi-spherical. Consider a set of $n$ cells, which are assumed to be hemi-spherical, on a flat twodimensional substrate, denoted by $\Omega \subset \mathbb{R}^{2}$. Each cell can be in two states: viable or dead, as two discrete states. Each viable cell is allowed to move and exerts a traction force on the substrate. By exerting this force, each cell releases a mechanical signal that is transmitted over the substrate. This force, giving a stress (force per area), results in small deformations of the substrate, hence there will be a (small) strain. Combination gives the mechanical energy, which is the strain energy density (energy per unit of volume). The strain energy density represents a scalar number, and no vectorial or tensorial quantity, and can therefore be evaluated and used easily as a transferred and detected signal through the substrate. Let $M_{i}^{0}$ denotes the strain energy density due to the exertion of the force $F_{i}$, at the very position of the cell $i$, then, assuming linear elasticity and small deformations of the substrate, we 
compute the strain energy density at the cell center by

$M_{i}^{0}=\frac{1}{2} \sigma \epsilon=\frac{1}{2} E_{S}\left(\mathbf{r}_{i}\right) \epsilon^{2}$,

where $\epsilon$ represents the strain of the substrate at the center of the cell, which is projected onto the substrate, and $E_{s}\left(r_{i}\right)$ represents the local elasticity modulus of the substrate. Since the substrate properties may vary over the domain $\Omega$, it is a function of the position of the center of cell $i$ projected onto $\Omega$, which is denoted by $\mathbf{r}_{i}$. Let $L$ and $d$, respectively, denote the thickness and local vertical displacement of the substrate caused by the upward pulling force of the cell on the substrate, we calculate the strain by

$\epsilon=\frac{d}{L}$,

and hence the strain energy density function can be written as

$M_{i}^{0}=\frac{1}{2} E_{S}\left(\mathbf{r}_{i}\right)\left(\frac{d}{L}\right)^{2}$.

Assuming low strains, Hooke's Law is applied to give

$\epsilon=\frac{1}{E_{S}\left(\mathbf{r}_{i}\right)} \frac{F_{i}}{A}=\frac{1}{E_{S}\left(\mathbf{r}_{i}\right)} \frac{F_{i}}{\pi R^{2}}$,

where $A$ and $R$, respectively, denote the area of the bottom of the cell on the substrate and the cell radius. Combining these equations, we get

$M_{i}^{0}=\frac{1}{2 \pi} \frac{F_{i}}{E_{S}\left(\mathbf{r}_{i}\right) R^{2}}, \quad$ for $\quad i \in\{1, \cdots, n\}$.

In the present study, we assume that the cell traction force, $F_{i}$, is equal to the maximum effective upward traction force exerted by a viable cell, $\hat{F}$ if cell $i$ is viable. Since dead cells do not exert any traction force, we assume that if cell $i$ is not viable, then its cell traction force is zero. In other words, we have the following discrete relationship:

$F_{i}=\left\{\begin{array}{l}\hat{F}, \text { if cell } i \text { is viable; } \\ 0, \text { if cell } i \text { is dead. }\end{array}\right.$

In our current simulations, all cells are assumed to be of the same phenotype, hence $\hat{F}$ is the same for all cells. A future extension of the model is to include several phenotypes, which means that various values of $\hat{F}$ will be used. In the present study, we assume that the cell traction force, $F_{i}$, is a measure for the viability of the cell. The above expression relates the signal exerted by cell $i$ to its viability. The exact analytical solution of displacements, strains, and stresses in an elastic layer with finite thickness (i.e. a substrate) that is loaded by a normal point force (i.e. by an attached cell), as assumed herein, was obtained by Burmister (1945a,b,c) over 60 years ago based on the earlier work of Boussinesq (1885), who studied a simpler problem —of an elastic half space loaded by a normal point force. The complete solution of the Burmister theory for a case of an elastic layer attached to a rigid foundation - which is suitable for describing most cell culture experiments-has been provided by Merkel et al. (2007). The analytical form in Merkel et al. (2007) requires integral solutions of Bessel functions and hence, will not fit numerical implementation for simulations of the motility of a large number of cells. Fortunately however, the displacement field induced by such a point force can be approximated to decay exponentially, as indeed noted by Merkel et al. (2007) in their Eq. (13). Therefore, at any location $\mathbf{r} \in \Omega$, we use the following expression to compute the transmitted signal as a result of the upward traction exerted by cell $i$

$M_{i}(\mathbf{r})=M_{i}^{0} \exp \left\{-\lambda_{i} \frac{\left\|\mathbf{r}-\mathbf{r}_{i}\right\|}{R}\right\}$,

for $\mathbf{r} \in \Omega, i \in\{1, \ldots, n\}$,

where $\mathbf{r}_{i}$ represents the location of cell $i$, projected on $\Omega$, further $\lambda_{i}$ is measure of how much the signal is attenuated, where we have

$\lambda_{i}=\frac{E_{s}\left(\mathbf{r}_{\mathbf{i}}\right)}{E_{i}}$.

The use of the exponential relation for the strain energy density is also supported by the finite-element solution shown in Fig. 1. Temperal dependencies are not considered in the present study, although this can be done in a straightforward manner. Further, $E_{i}$ denotes the elasticity modulus of cell $i$. Since energy is a scalar quantity, it is additive, and hence for the collection of $n$ cells, we get the following total strain energy density at position $\mathbf{r}$

$$
\begin{aligned}
& M(\mathbf{r})=\sum_{j=1}^{n} M_{j}(\mathbf{r})=\sum_{j=1}^{n} M_{j}^{0} \exp \left\{-\lambda_{j} \frac{\left\|\mathbf{r}-\mathbf{r}_{j}\right\|}{R}\right\}, \\
& \quad \text { for } \mathbf{r} \in \Omega .
\end{aligned}
$$

This above simplification of the strain energy density function is motivated in Fig. 1, where a finite-element plot of the strain energy density function is displayed. Further, we display the line plot. The solution that is displayed holds for one cell only and note that this figure only displays a visual motivation for the use of an exponentially decaying strain energy density function.

Let $\mathbf{r}_{i}(t)$ represents the location of the center of cell $i$ (projected onto $\Omega$ ) at time $t$, then by the use of the above expression, we calculate the mechanical stimulus that is sensed by cell $i$, in which also its own signal is incorporated by

$$
\begin{aligned}
M\left(\mathbf{r}_{i}\right)= & \sum_{j=1}^{n} M_{j}\left(\mathbf{r}_{i}\right)=\sum_{j=1}^{n} M_{j}^{0} \exp \left\{-\lambda_{j} \frac{\left\|\mathbf{r}_{i}-\mathbf{r}_{j}\right\|}{R}\right\} \\
= & M_{i}^{0}+\sum_{j=1_{j \neq i}}^{n} M_{j}^{0} \exp \left\{-\lambda_{j} \frac{\left\|\mathbf{r}_{i}-\mathbf{r}_{j}\right\|}{R}\right\} \\
& \text { for all } i \in\{1, \ldots, n\} .
\end{aligned}
$$




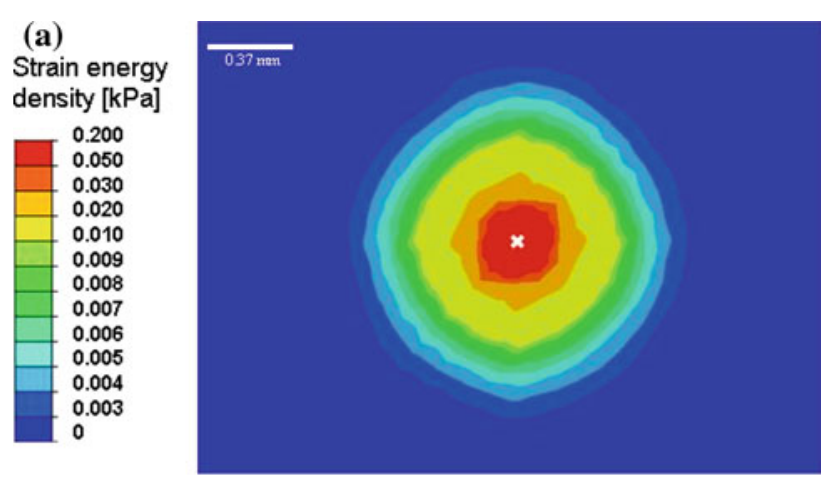

(b)

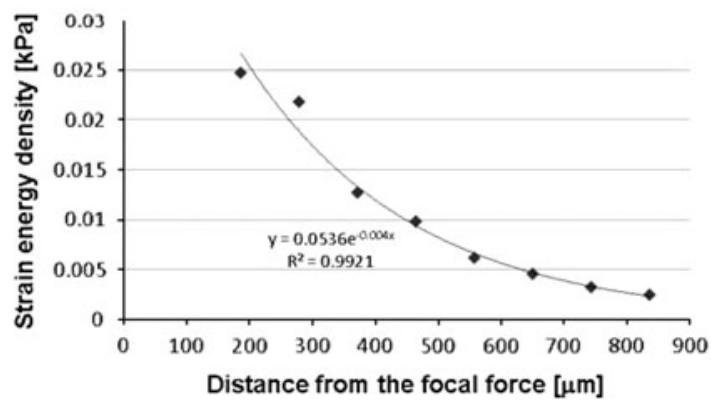

Fig. 1 Finite-element solutions of the strain energy density around a single cell

Next, we consider the displacement of each cell $i$ as a result of sensing the mechanical signal. It is assumed that the displacement vector over a time frame $\Delta t$ is a linear combination of all the unit vectors connecting cell $i$ to all other cells $j \in\{1, \ldots, n\} \backslash i$. The weight factor for each pair $(i, j)$, $j \neq i$, is determined by the sensed mechanical stimulus, being the strain energy density function and the viability of the cell. In a fully deterministic formalism for cell movement, the displacement of cell $i$ over a time frame $\Delta t$ is parallel to

$\mathbf{z}_{i}=\sum_{j=1}^{n} M_{j \neq i}\left(\mathbf{r}_{i}(t)\right) \mathbf{v}_{i j}(t), \quad$ for all $i \in\{1, \ldots, n\}$,

where $\mathbf{v}_{i j}$ represents the unit vector connecting cell $i$ to cell $j$, which is given by

$\mathbf{v}_{i j}=\frac{\mathbf{r}_{j}-\mathbf{r}_{i}}{\left\|\mathbf{r}_{j}-\mathbf{r}_{i}\right\|}$

and this vector is mapped onto zero if $\left\|\mathbf{r}_{j}-\mathbf{r}_{i}\right\|=0$. We will use the unit vector of $\mathbf{z}_{i}$, given by

$\hat{\mathbf{z}}_{i}=\frac{\mathbf{z}_{i}}{\left\|\mathbf{z}_{i}\right\|}$

Since the displacement over a time frame is assumed to be in the direction of $\mathbf{z}_{i}$ where the magnitude of the displacement is assumed to be proportional to the strength of the mechanical signal, we have

$\mathbf{r}_{i}(t+\Delta t)-\mathbf{r}_{i}(t)=\Delta t \alpha_{i} M\left(\mathbf{r}_{i}\right) \hat{\mathbf{z}}_{i}$,

for all $i \in\{1, \ldots, n\}$,

where $\alpha_{i}$ is a parameter with a dimension $\left[\frac{m^{2} s}{k g}\right]=\left[\frac{m^{3}}{N s}\right]$, in which the force is directed along $\Omega$, hence perpendicular to the upward cell traction force $F$. This cell-substrate friction force directed along $\Omega$ is denoted by $f$. The parameter $\alpha_{i}$ should also contain the cell viability since the cell mobility depends on the cell viability. Therefore, we express $\alpha_{i}$ by

$\alpha_{i}=\left(\frac{F_{i}}{\hat{F}}\right)^{2} \beta_{i} \frac{R^{3}}{f}$.

Here, $\hat{F}$ as mentioned earlier represents the effective upward traction force exerted by a viable cell, and $\hat{F}$ is a property of the specific phenotype of the cell. This quantity may be different for each phenotype. The cell-substrate friction effectively represents the averaged contribution of focal adhesions along the entire base of the cell without considering each localized connections of intergrins. This force is related to the traction force by

$f=\mu F_{i}$,

where $\mu$ denotes the cell friction coefficient, and following Gefen (2010), this dimensionless quantity is set to $\mu=0.2$. Furthermore, $\beta_{i}$ with unit $s^{-1}$ quantifies the mobility of the cell surface of a viable cell. Hence, the parameter $\alpha_{i}$ is given by

$\alpha_{i}=\frac{\beta_{i} R^{3}}{\mu \hat{F}^{2}} F_{i}$.

Hence, we obtain the following expression for the change in position of cell $i$

$\mathbf{r}_{i}(t+\Delta t)-\mathbf{r}_{i}(t)=\Delta t \frac{\beta_{i} R^{3}}{\mu \hat{F}^{2}} F_{i} M\left(\mathbf{r}_{i}\right) \hat{\mathbf{z}}_{i}$,

for all $i \in\{1, \ldots, n\}$.

Since $M_{j}$ is linearly proportional to $F_{j}$, it can be seen that the motion of cell $i$ depends on its own viability, but also on the viability of the other cells. This is consistent with what one would expect on forehand: The mechanical signal that is sensed by cell $i$ depends on the traction forces exerted by the other cells. The magnitude of the traction force exerted by a cell $j$ depends on its viability. The hypotheses so far imply that the model predicts that if just two viable cells are present, then they will always receive the signals that are transmitted due to pulling forces exerted by the cells. Once they receive each others signals, then they will move toward each other at a speed that is determined by their mobility, diameter, friction coefficient, and viability. The signal strength, from the other (target) cell a cell would move to, hardly influences the cell 
Table 1 Input data

\begin{tabular}{lll}
\hline Quantity & Value & Unit \\
\hline$E_{S}$ & 5 & $\mathrm{kPa}$ \\
$E_{c}$ & 0.5 & $\mathrm{kPa}$ \\
$R$ & 0.04 & $\mu \mathrm{m}$ \\
$\mu$ & 0.2 & - \\
$\hat{F}$ & 1 & $\mathrm{nN}$ \\
$p$ & 0.01 & - \\
$q$ & 0.05 & - \\
$p_{m p}$ & 0 & - \\
$\beta_{i}$ & 10 & $\mathrm{~s}^{-1}$ \\
\hline
\end{tabular}

velocity. This advocates for the introduction of a detection threshold for the strain energy density as a minimum signal strength that a cell can only detect. If one would use a detection threshold of $\varepsilon$, then this means from Eq. (6), that signal strengths that are detected by a cell satisfies

$M_{i}(\mathbf{r})=M_{i}^{0} \exp \left\{-\lambda_{i} \frac{\left\|\mathbf{r}-\mathbf{r}_{i}\right\|}{R}\right\} \geq \varepsilon$

Hence, this gives for the maximum distance for a cell to be able to detect the signal from cell $i$ :

$\left\|r-r_{i}\right\| \leq \frac{R}{\lambda} \ln \left(\frac{M_{i}^{0}}{\varepsilon}\right)$.

Reinhart-King et al. (2008) were, as far as we know, the first authors to evidence cell-cell communication via mechanical forces through compliant substrates. They found for elasticity moduli of substrate and cell of, respectively, approximately $5 \mathrm{kPa}$ and approximately $0.5 \mathrm{kPa}$ that the maximum distance over which cells sense each other is about $\hat{d}=30 \mu \mathrm{m}$. Hence, the detection threshold is defined by

$\varepsilon=M_{i}^{0} \exp \left\{-\lambda_{i} \frac{\hat{d}}{R}\right\} \approx 2.7 \cdot 10^{-31}$

using data from Table 1 (see Sect. 4 in this manuscript). In the spatial dimensions that we use in the current study, the signal strength always exceeds the detection threshold. We assume that the cells will react to each response that they detect and that their velocity of motion only depends on the parameters of the individual cell and cell-substrate interaction. Of course, in the case of many cells, the cells will react most strongly to the signals received from the nearest cells.

In the next subsections, we will outline the incorporation of cell death and cell proliferation as stochastic processes and the repulsive elastic contact forces between cells as they impinge each other.
2.1 Incorporation of cell death and proliferation as stochastic processes

All probabilities that we consider are normalized to unity. Since the cell viability of cell $i$ is quantified by $F_{i}$, we use $F_{i}$ to account for possible cell death by setting $F_{i}=0$ if cell $i$ has died. At each time frame, the likelihood for each cell to die is assumed to be given by $p$. In our calculations, we use $p=0.01$ as a hypothetical value. This probability may be linked to the condition of the cell culture or its environment like temperature, toxicity, and $\mathrm{pH}$. Let $x_{i}$ be the random variable to account for the possible death of cell $i$, then we set $x_{i}=\operatorname{rand}(1)$ and if $x_{i}>0.99$, then $F_{i}=0$. This procedure is performed for all cells $i \in\{1, \ldots, n\}$. In the present study, we neglect cell degradation after cell death, hence cell death is a sudden, discrete transition, or state in our model. We assume that the dead cell does not exert any traction on the substrate and it does not move. Despite the release of chemicals during the process of dying of a cell, and since we only consider mechanical influences on cellular motion and hence neglect all chemical relations in the current study, we assume that the dying process suddenly removes all the influence that a dying cell has on the motion of the other cells. Furthermore, it stays at the position where it died. This position cannot be occupied by the viable cells.

Next, we introduce cell proliferation into the model as a stochastic process. We denote the probability of a cell to generate a daughter cell by $q$, for which we use a hypothetic $q=0.05$ in our simulations. We realize that the used probabilities $p$ and $q$ can only be estimated using experimental studies. We did not find a source from literature for these values. Suppose that during the time interval $[t, t+\Delta t]$, cell $i$ divides into two cells. Then from Eq. (17), the displacement of cell $i$ is computed. Subsequently, we assume that the positions of mother cell $i$ and its daughter cell are such that their center of mass coincides with the position of the original mother cell $i$ as was computed by the use of Eq. (17). The positions of the mother cell and daughter cell are such that they are shifted at a random orientation to the displacement vector with an additional displacement of magnitude $R$. We realize that this is a major simplification since the actual orientation is a stochastic variable as well. However, Hoehme and Drasdo (2010) model the separation of a cell into two daughter cells by a stochastic process using a Langevin equation. This will be dealt with in a more rigorous way in future studies. Further, we intend to deal with cell differentiation as a stochastic process as well in future studies.

\subsection{Incorporation of randomness in cellular motion}

We also study the option that the motion of a cell is (partly) random. At each time frame, there is a probability that the cell motion differs from the motion that it should have according 


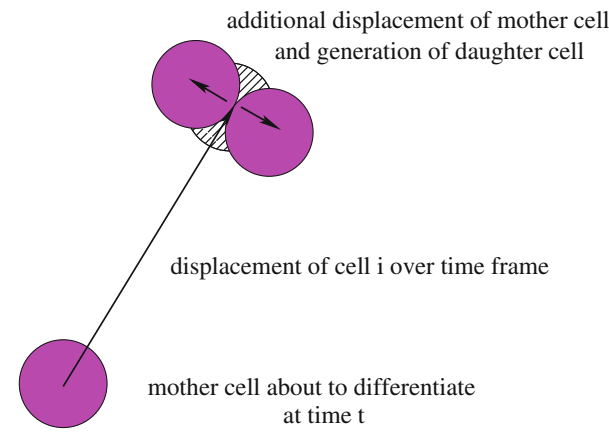

Fig. 2 A schematic of the movement of a mother cell and generation of a daughter cell. The shaded image is the phantom cell that followed the displacement from Eq. (17). The other cells have undergone an additional displacement with magnitude $R$ and perpendicular to the displacement of the phantom cell

to the previous section. Then, a randomizer gives the angle of the deviation, if the cell is supposed to move in a random direction. The length of movement at the time frame is determined by the cell viability only. In the current model, a complete arbitrary movement of cells is admissable. Then, the only thing that is fixed is the length of movement. Mathematically, this is incorporated as follows: Assume that there is a possibility that a cell does not respond to the mechanical stimulus, at a certain time (or certain time interval). Then, the cell movement is given by

$$
\begin{aligned}
& \mathbf{r}_{i}(t+\Delta t)-\mathbf{r}_{i}(t)= \\
& \quad \Delta t \alpha_{i} u_{i} M\left(\mathbf{r}_{i}\right) \hat{\mathbf{z}}_{i}+\Delta t\left(1-u_{i}\right) \mathbf{w},
\end{aligned}
$$

where $u_{i}:[0,1] \rightarrow\{0,1\}$ is a stochastic variable, and $\mathbf{w}$ is a stochastic vector (by using the angle of orientation, with respect to its usual pathway without any stochastic perturbation). The stochastic variable $u_{i}$ represents the insensitivity of cell $i$ with respect to the movement as dictated by the strain energy density, and it is determined by the random variable $y_{i}$, such that

$u_{i}=u_{i}\left(y_{i}\right)=\left\{\begin{array}{l}1, \text { if } y_{i} \in\left[0,1-p_{m p}\right] \\ 0, \text { if } y_{i} \in\left(1-p_{m p}, 1\right] .\end{array}\right.$

\subsection{Contact forces between impinging cells}

Next, we incorporate the contact forces between elastic impinging cells, by following the principles outlined in Gefen (2010) (Fig. 2). These contact forces will act as repulsive forces so that the centers of adjacent cells are not allowed to overlap. This effect is due to the linear elastic deformation of the cell bodies. To derive the resulting contact force, that is the invagination force, Gefen (2010) uses the assumption of two contacting elastic spheres. The cells come first come into point contact with each other. Using standart principles from contact mechanics, see for instance Johnson (1985), the

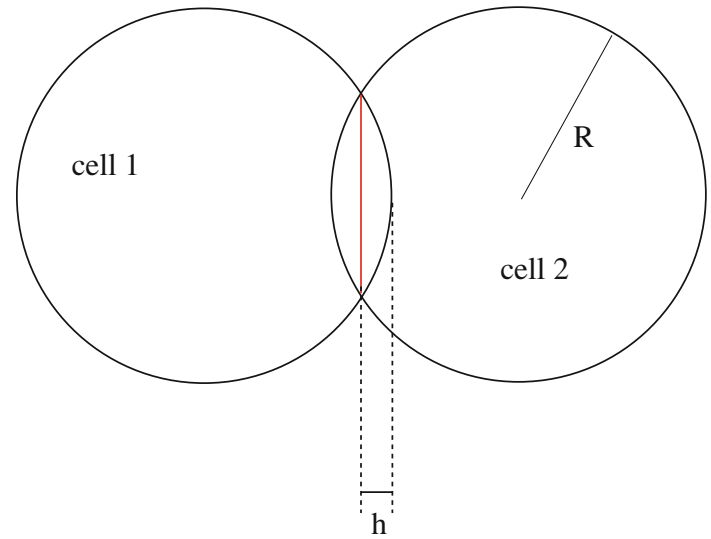

Fig. 3 Two impinging cells

combined radius and reduced elastic modulus can be derived. As the cells collide more into each other, the invagination force builds up. From contact mechanics Johnson (1985), it follows that the resulting repulsive force can be determined from the relation between the indentation $h$, see Fig. 3, and contact radius. This gives the following resulting force, $\phi$, is given by

$\phi=\frac{4}{3} \sqrt{h^{3}\left(E^{*}\right)^{2} R^{*}}$,

where $R^{*}=\frac{R}{2}$ and $E^{*}=\frac{2}{3} E$.

The work done is given by

$W=\int_{0}^{h} \phi(\bar{h}) \mathrm{d} \bar{h}=\frac{8}{15} \sqrt{R^{*}} E^{*} h^{\frac{5}{2}}$.

To get the strain energy density $M^{i j}$, we divide by the volume of the cell, being $\frac{4}{3} \pi R^{3}$, which gives

$M^{i j}=\frac{6}{15} \frac{\sqrt{R^{*}} E^{*} h^{\frac{5}{2}}}{\pi R^{3}}$

The final result for the total strain energy density function becomes

$\hat{M}_{i}(\mathbf{r})=M_{i}(\mathbf{r})-M^{i j}$,

where $\hat{M}_{i}$ and $M^{i j}$, respectively, denote the total strain energy density and the contribution to the strain energy density from the elastic interaction between neighboring cells. This quantity should be seen as an energy relative to some fixed energy level or as a potential in order to allow it to have negative values.

\section{Analytic solution for two cells}

For the case of two cells without cell death, without cell division, and without randomness in the motion, we derive the 
analytic solution, which is valid as long as the cells do not yet impinge. For two cells, we use the following simplifying hypotheses:

$-\lambda_{1}=\lambda_{2}=\lambda \in \mathbb{R}$

- $M_{1}^{0}=M_{2}^{0}=M \in \mathbb{R}$

- $\alpha_{1}=\alpha_{2}=\alpha \in \mathbb{R}$.

Here, the parameters $\alpha, \lambda$, and $M$ are obtained by the use of the values given in Table 1, using the relations in Sect. 2 . Note that the problem has not been made non-dimensional. Then, one easily obtains

$$
\left\{\begin{array}{l}
\mathbf{r}_{1}^{\prime}=\alpha M \cdot\left[1+\exp \left\{-\lambda \frac{\left\|\mathbf{r}_{1}-\mathbf{r}_{2}\right\|}{R}\right\}\right] \cdot \frac{\mathbf{r}_{2}-\mathbf{r}_{1}}{\left\|\mathbf{r}_{1}-\mathbf{r}_{2}\right\|}, \\
\mathbf{r}_{2}^{\prime}=-\mathbf{r}_{1}^{\prime} .
\end{array}\right.
$$

This implies that $\mathbf{r}_{1}^{\prime}+\mathbf{r}_{2}^{\prime}=0$, and hence $\mathbf{r}_{1}+\mathbf{r}_{2}=2 \mathbf{r}_{c}$, where $\mathbf{r}_{c}$ denotes the center of mass, which does not move. Defining $r:=\left\|\mathbf{r}_{c}-\mathbf{r}_{1}\right\|$, gives the following differential equation

$\frac{\mathrm{d} r}{\mathrm{~d} t}=-\alpha M\left[1+\exp \left\{-2 \lambda \frac{r}{R}\right\}\right]$.

Integration of the above equation gives

$$
\begin{aligned}
& r(t)= \\
& \frac{R}{2 \lambda} \ln \left[\exp \left\{-2 \frac{\lambda}{R} \alpha M t\right\}\left(1+\exp \left\{2 \frac{\lambda}{R} r_{0}\right\}\right)-1\right],
\end{aligned}
$$

where $r_{0}$ denotes the initial distance from the cells to the center of mass. The actual intercellular distance is obtained by multiplying the above equation by 2 . In Fig. 4, we plot the analytic solution and the numerical solution. It can be seen that the agreement is excellent, in particular at the early stages. This gives confidence in our numerical solutions. The oscillatory behavior of the numerical solution is due to a struggle between repulsive and attractive forces as the cells come into close contact. The input parameters that were used in these computations are listed in Table 1.

Furthermore, it is possible to determine the time at which two cells impinge, $t_{I}$, by

$t_{I}=\frac{R}{2 \lambda \alpha M} \cdot \ln \left[\frac{1+\exp \left\{\frac{2 \lambda r_{0}}{R}\right\}}{1+\exp \{2 \lambda\}}\right]$.

From the above equation, it is possible to show that $t_{I}$ has a limit value as $\lambda \rightarrow \infty$ :

$$
\lim _{\lambda \rightarrow \infty} t_{I}(\lambda)=\frac{R\left(\frac{r_{0}}{R}-1\right)}{\alpha M},
$$

which will act as a horizontal asymptote, and hence the dependence on $\lambda$ vanishes as $\lambda$ gets 'very large'. We have to realize that the analytic solution is only an approximation to the real simulations.

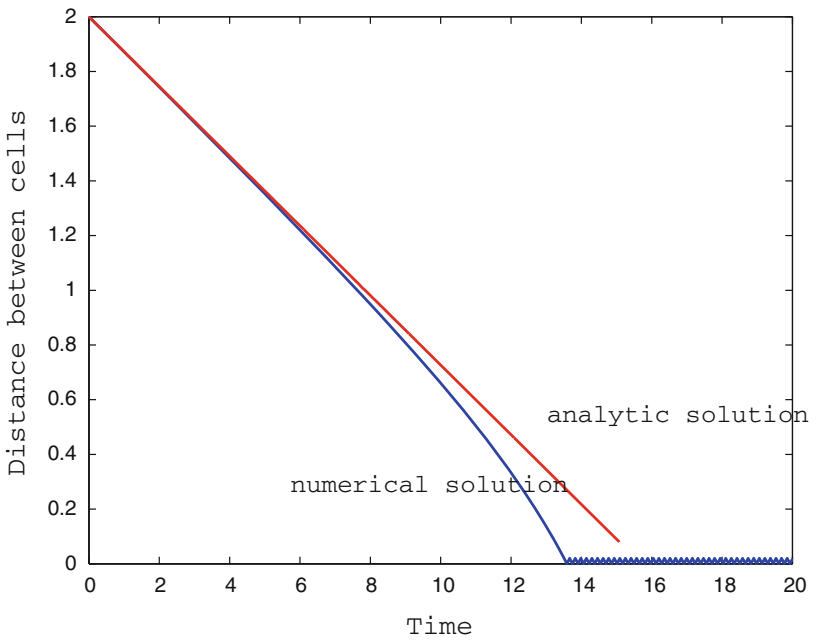

Fig. 4 The distance between two cells as a function of time for both the analytic and the numerical solution

\section{Applications}

In this section, we consider several applications of the model. First, we deal with a two-cell system, which was compared with the analytic solution in the previous section. Subsequently, we deal with a situation that mimics healing of an epidermal wound. We consider a domain of cells in which an artificial wound is created by removing the cells in the center of the domain of computation. Subsequently, we consider a couple of cells in the center of the domain of computation and track the gradual invasion of the cells with mechanisms like movement and cell division into the rest of the domain of computation. This case could be seen as a simplification of modeling tumor growth or organ formation. Note that cell differentiation is not taken into account in this formalism, which should be done when organ development is modeled. Lemmon et al. (2009) studied the cell traction forces and found that these forces were of the order of a nano-Newton. Wang and Lin (2007) describe several experimental techniques to obtain cell traction forces. In the study by Califano and Reinhart-King (2010), cellular traction forces are determined by the combination of experiments and regression procedures. We use the input data from Table 1, unless stated otherwise.

As a numerical method, we use a second-order accurate explicit Runge-Kutta method (Heun's method) for the time integration, with an adaptive time step determined by the cell with the highest velocity. The non-linear nature of the problem to determine the cell positions makes an implicit method less attractive due to the need of an inner iteration. Due to the repulsing forces which result from hard impinging cells, the time step needs to be relatively small sometimes. We choose 


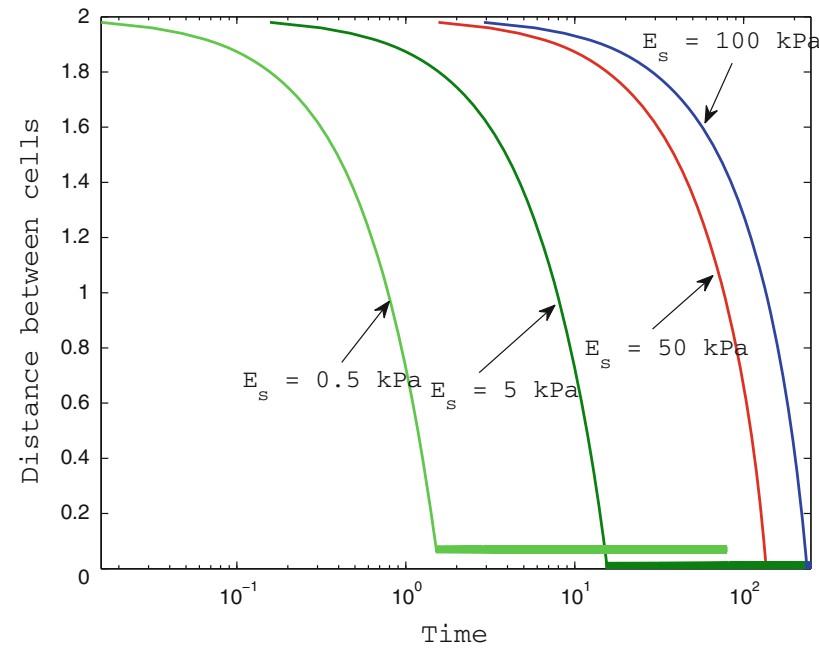

Fig. 5 The distance between two cells as a function of time for several stiffnesses of the substrate. The unit is in $\mathrm{kPa}$

to use

$\Delta t=\frac{R}{2 \cdot \max _{i \in\{1, \ldots, n\}}\left\|v_{i}\right\|}$.

This will guarantee that the cells are not displace over a larger distance than $25 \%$ of their diameter. Reducing the time step, by taking half of the present time step, did not alter the results, which means that convergence is accomplished.

\subsection{A two-cell problem}

First, we consider two cells. The cells are positioned on the points $(-1,0)$ and $(1,0)$. We track the distance as a function of time. In this section, we change the elasticity modulus of the substrate and the probability that motion proceeds in a random way.

\subsubsection{The elasticity of the substrate}

Figure 5 shows the results of variation of the Young's modulus of the substrate. It can be seen that a stiffer substrate gives less attenuation of the signal and hence the cells feel the presence of the surrounding cells less, which is consistent with the experimental findings of Haga et al. (2005) in epithelial cells cultured on collagen gel versus glass substrates. Therefore, the velocity of the cells decreases as the substrate becomes stiffer, a result which is supported by experiments of Lo et al. (2000) who found that the speed of fibroblasts was 1.7 times greater on substrates with stiffness of $E_{S}=140 \mathrm{kdyn} / \mathrm{cm}^{2}$ with respect to their speed on substrates with $E_{S}=300 \mathrm{kdyn} / \mathrm{cm}^{2}$. This is clearly visible in Fig. 5. At the final times, as the cells approached each other maximally, an oscillating behavior arises. This is due to the repulsive force that is experienced as the cells are located at a distance that is too small. Then, the cells move away from each other. Subsequently, the cells are separated, and then the cells move toward each other. A smaller time step could decrease this behavior, but these oscillations can never be removed completely. It is questionable whether these oscillations could not be there, since each cell may have a non-zero reaction time. If this is the case, then the time step should be chosen at least larger than the reaction time of a cell in which this reaction time may introduce a delay into the model. Here, the viability could also play a role. In the study by Reinhart-King et al. (2008), it is observed that cells communicate through the substrate. Cells that are separated at distances of less than approximately $30 \mu \mathrm{m}$, will move to each other. Whereas if cells come into a very close contact, they may either climb on top of each other (or climb over each other) or repel from one another. It is clear that we assume that cells do not climb over each other, but that they stay in contact with the substrate and hence, they exert and experience a repulsive force as they impinge on each other. Since, we use the cell viability as a discrete parameter, and we omit the influence of a possibly continuous cell viability. This implies that a cell is either fully viable or dead. Finally, we remark that using a time step of half the present time step does not significantly change the simulation results. Using a very large time step (of about ten times as large) gives a stronger oscillating behavior when the cells are in very close contact.

\subsubsection{Allowing randomness in motion}

Subsequently, we allow a certain degree of randomness in the motion of the cells. We plot the results for various levels of likelihood that the motion of a cell is perturbed. In this study, we assume that the magnitude of the cell displacement at each time step is solely influenced by the mechanical stimulus that the cell experiences from its neighbor(s). The results are plotted in Fig. 6. It can be seen that despite the probability that a cell moves toward its direction as solely dictated by the mechanical stimulus can be small, the cells will cluster in the end. Further, we show the evolution of the cellular paths in the course of time. Due to random motion, the cells keep on moving (as dictated by the mechanical, being attractive and repulsive forces, stimulus). This is visible in Fig. 7. The randomly perturbed movement causes a certain direction toward which a cell moves. The attracting 'force' dictates the probability of the other cells to follow the cell that just carried out a random movement. Since the probability of perturbed movement is relatively small, the cells will stay close to each other and 'move like a dancing pair', under repulsion and attraction. In the left figure, we also show the path of motion in the case of no randomness. Then, the cells move to each other in a straight line, as expected. 


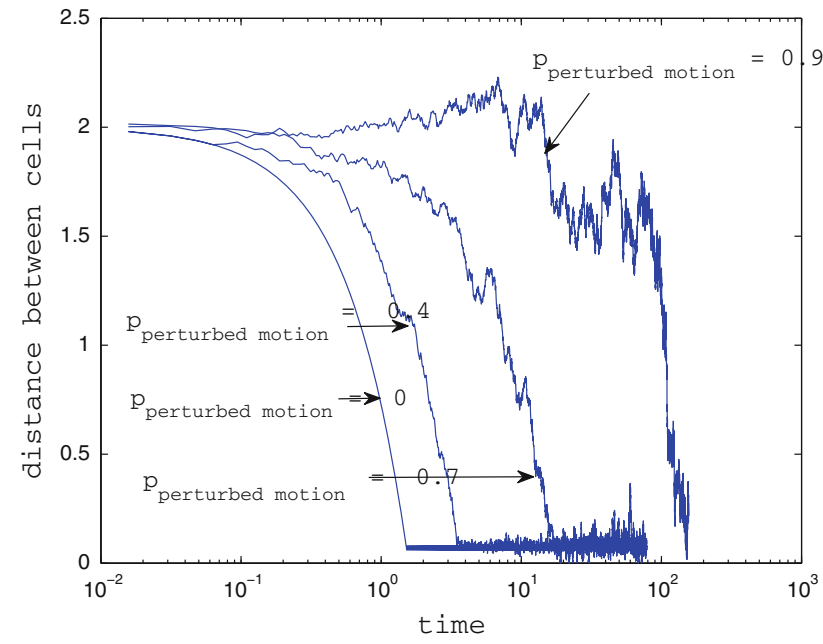

Fig. 6 The distance between two cells as a function of time for several values of the probability of perturbed direction of motion

\subsection{Artificial wound healing}

We consider a circular domain with radius $L=1$, in which the cells are initially arranged in an ordered way, see Fig. 8. In our basis set of parameters, we use $E_{\text {cell }}=5 \mathrm{kPa}$, and $E_{\text {gel }}=0.5 \mathrm{kPa}$, hence $\lambda=10$. If cells impinge on each other, they will experience and exert a repulsive force. We assume that cell motion is fully deterministic and that the probabilities of cell division and death are, respectively, given by 0.01 and 0.001 per time frame. The probabilities were chosen smaller to emphasize more on the cellular motion due to the mechanical conditions. Note that these values were taken as hypothetical. We also note that our simulation tool is also capable of dealing with random motion in cell colonies. However, since this perturbation does not alter the trends in the results, we decided to exclude randomness in motion in this simulation and to study this effect separately after some numerical analysis. Upon removing all cells within a circle

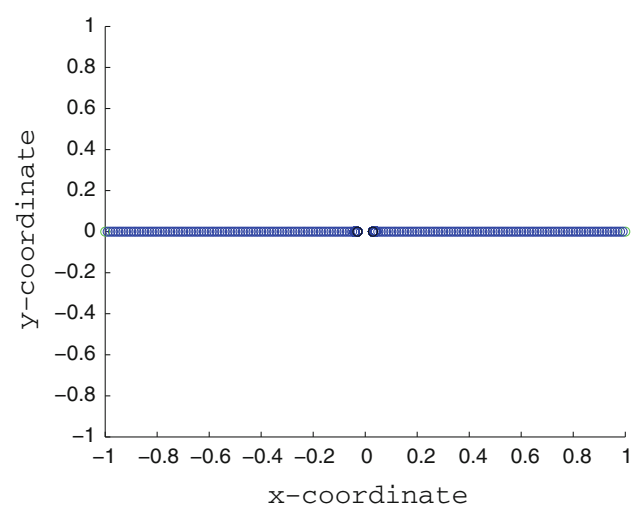

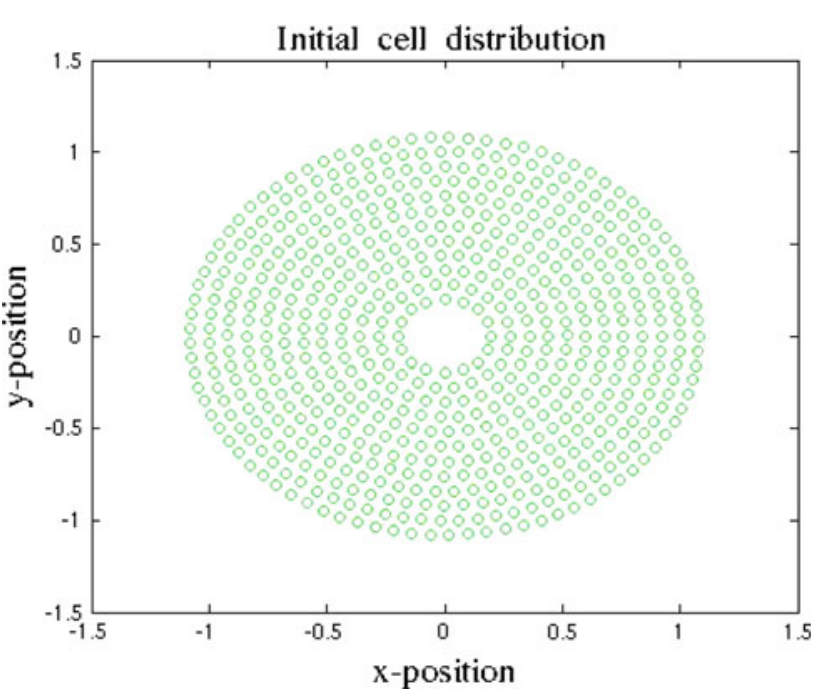

Fig. 8 The initial configuration of a mimicked wound

of a defined radius, we mimic the occurrence of a wound. The cells are confined within a circle with diameter $L$. A similar force is used to repel the cells from the outer boundary as in Sect. 2.3, where the wall is assumed to be rigid. The cells will move according to the earlier described mechanism, and they will proliferate and die. In Fig. 9, it can be seen that the entire gap, from which the cells were removed, starts to get filled with cells in the course of time. After 350 time steps, the wound is filled and the entire domain of computation is filled with cells. The model predicts a deviation from circular geometry of the gap as time proceeds. This is also observed in experimental studies, see the section on "Discussion".

\subsection{An invasive colony}

We use the set of basis variables as in the previous section, and we start with one cell at the origin. As time proceeds, we see the ingress of the cells further into the domain of computation as a result of migration and mitosis. The extension

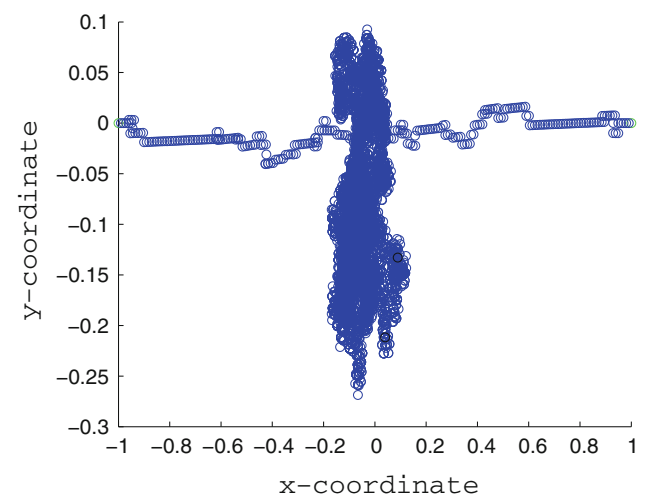

Fig. 7 The position of two cells as a function of time for two cases: Left no stochastic perturbation of motion; Right with stochastic perturbation of motion, see Table 1 
100

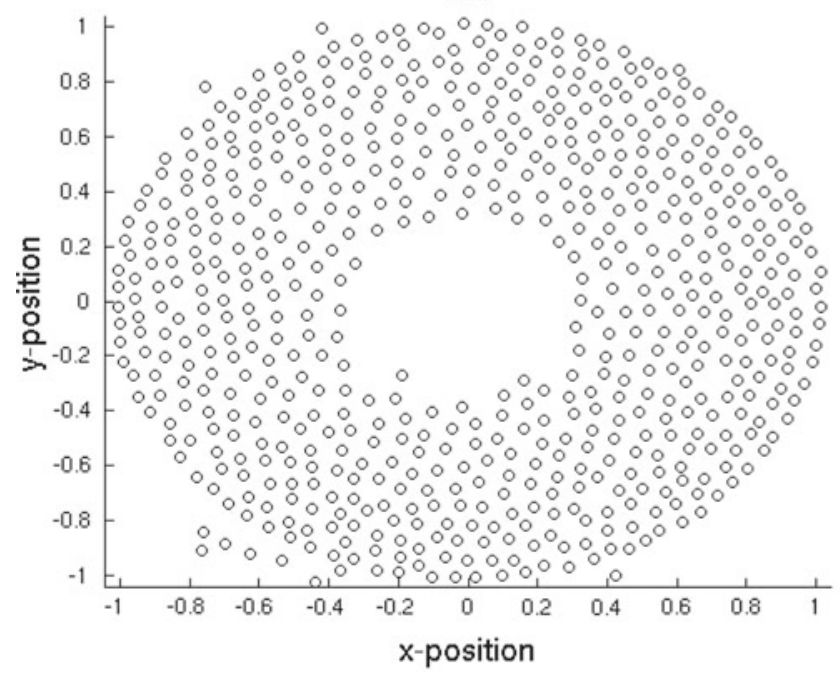

300

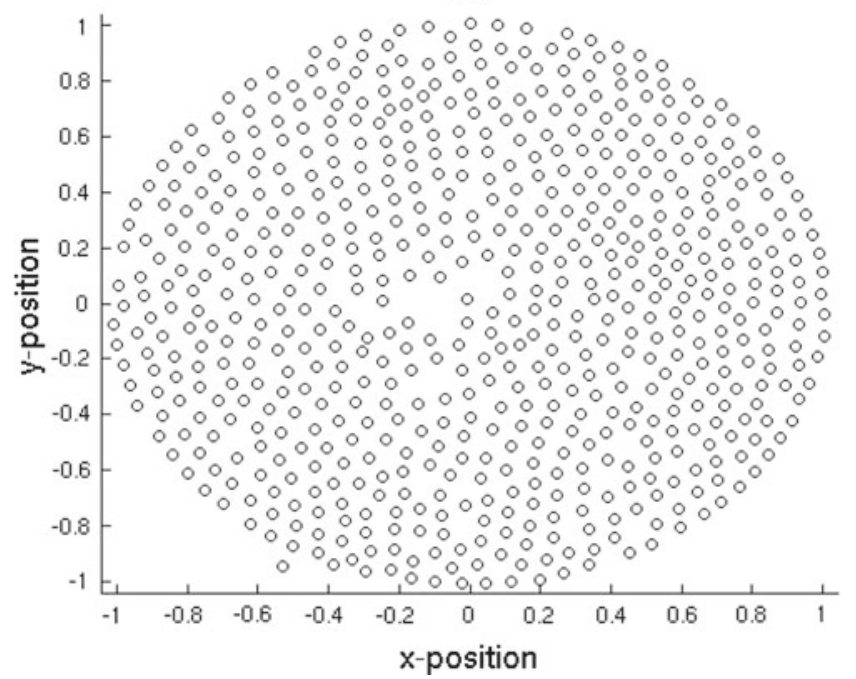

200

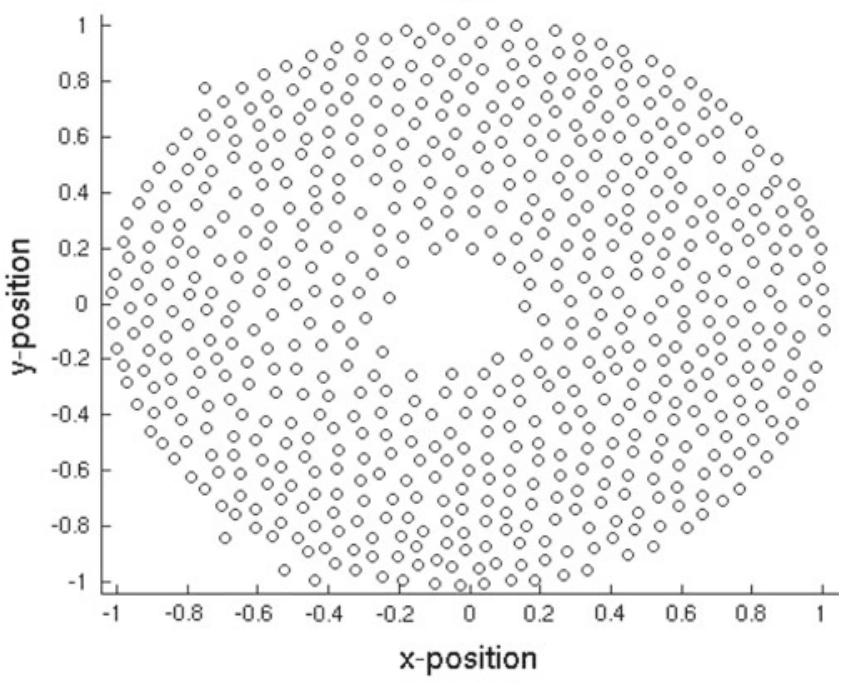

350

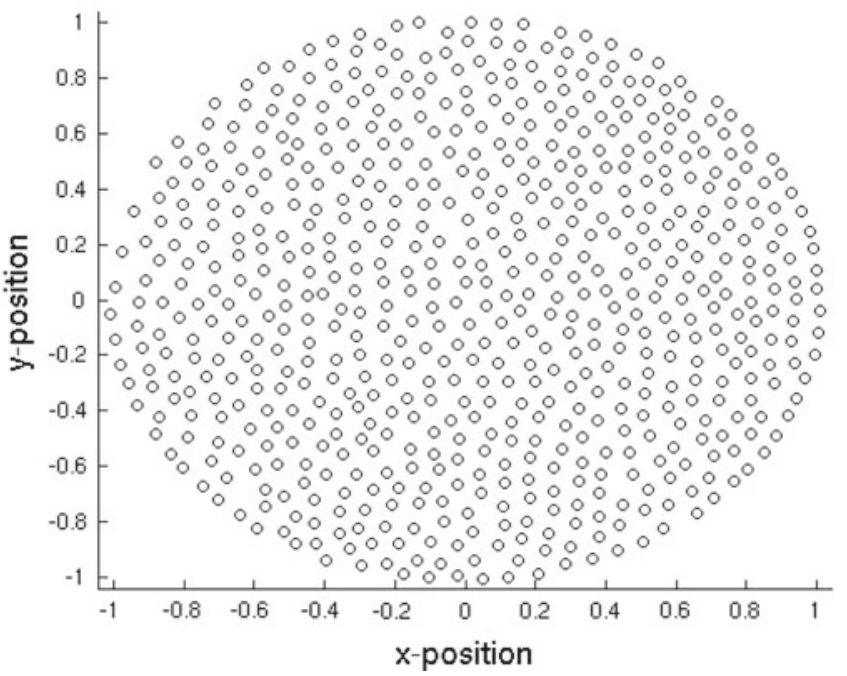

Fig. 9 Wound healing: The arrangement of the cells after 100, 200, 300, and 350 time iterations. In this simulation, we assumed that there is no stochastic perturbation of motion

of the imaginary cell culture proceeds in an almost circular fashion, see the plots in Fig. 10. Here, we also assumed that there is no stochastic perturbation of cellular movement and that the other probabilities of cell death and cell division are the same as in the previous subsection on artificial wound healing. As the cells have conquered the whole domain, the cells are rather packaged in the origin. As time proceeds further, we arrive at a more homogeneous distribution of the cells, see also Fig. 10.

\section{Discussion and qualitative experimental validation}

The model that we developed so far is capable of dealing with cell movement on a planar substrate, influenced by the mechanical properties of the cells and substrate. Cell death and cell proliferation are incorporated as stochastic processes. Furthermore, the motion of the cells is biologically a biased random process. This issue has been incorporated into the model as well. Due to genericity of the model, it is easy to account for cell differentiation too as a stochastic process. This feature can be built in easily, and the differentiated cells can be marked by having different cell properties, such as pulling force, rate of motion, cell death probability, and so on. In the present work, we consider a simplified domain in either a rectangular domain or a circular domain. This domain can be given any shape. The model will also allow to deal with several cell types that are moving around at the same time in the domain of computation. This step will be incorporated in the future. This will allow to apply 

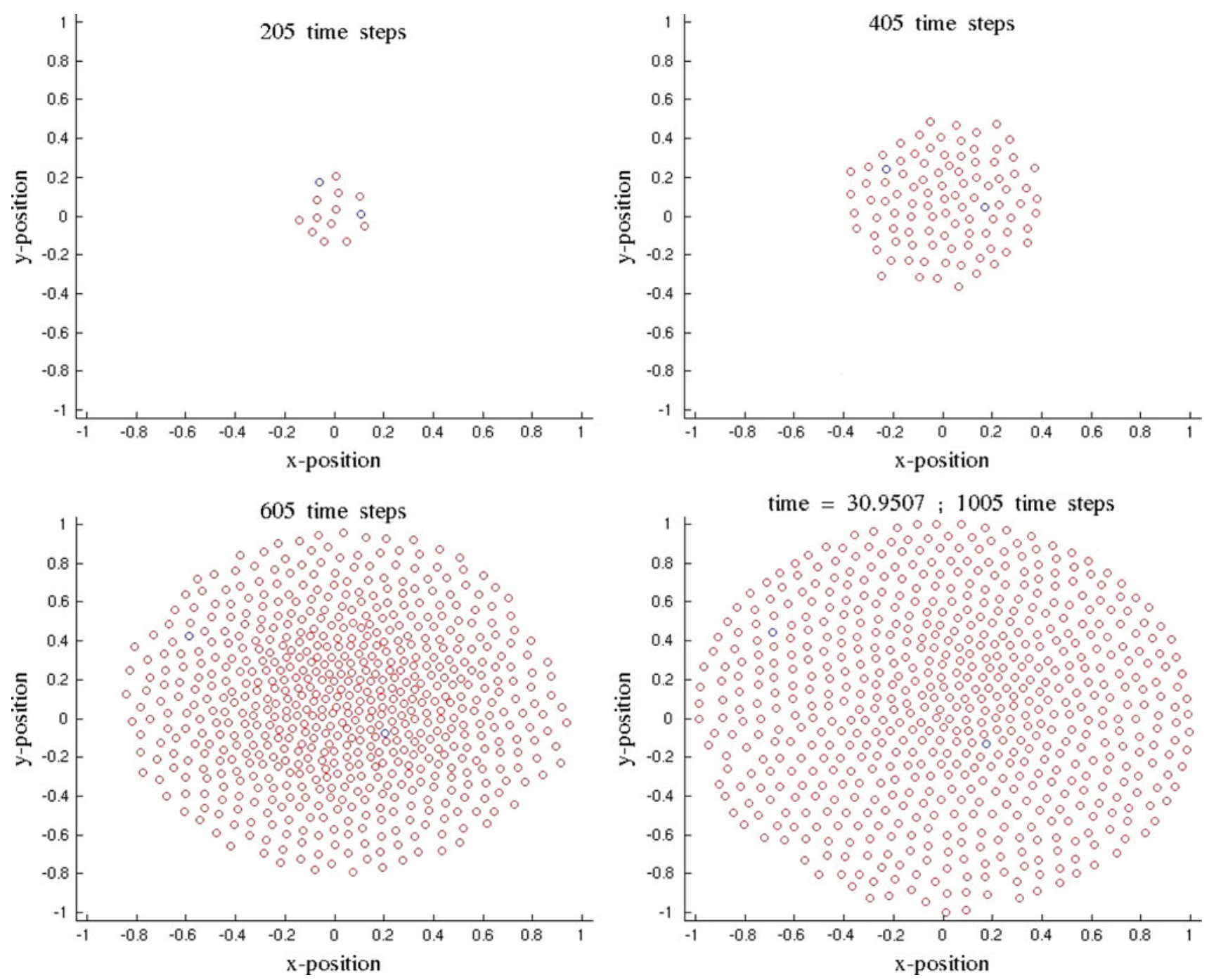

Fig. 10 Invasive colony: The arrangement of the cells after 205, 405, 605, and 1,005 time iterations. In this simulation, we assumed that there is no stochastic perturbation of motion

the model to growth of certain types of solid tumors, healing of epidermal wounds, or even organ development. It is also straightforward to extend the current approach to three spatial dimensions.

The model that has been constructed so far is preliminary and does not yet account for chemotaxis, a multi-phenotype approach, an-isotropies (directional dependencies), external mechanical forces, haptotaxis, toxicity. In the future, we plan to incorporate these phenomena and apply the model to processes like organ development and deterioration, and to more detailed wound healing or angiogenesis. Furthermore, we plan to compare the model results with simulation results obtained from partial differential equations based models.

The current model presents a continuous formalism, based on ordinary differential equations, to model the dynamics of cell migration, division, and death. This model is complementary to already existing models, either based on lattice principles or on partial differential equations. An advantage of the current with respect to partial differential equations is that it is possible to include stochastic events more easily and to follow individual cells. With respect to the lattice-based models, the current model treats the position of cells in a continuous way, rather than having cells at predefined allowable locations. A disadvantage of the current model, in particular with respect to partial differential equations, is that once the area of considered tissue is large, then the computation tend to become expensive since many cells are needed.

We use the following experimental setting as a qualitative validation of the current model. NIH/3T3 fibroblast cells from a mouse source (ATCC, www.atcc.org) were cultured in monolayers in 6 -well plates $(5 \times 104$ cells seeded per well $)$ in a medium containing Dulbecco's Modified Eagle's medium (with $4.7 \mathrm{~g} / \mathrm{l}$ Glucose) supplemented with $10 \%$ fetal bovine serum, $1 \%$ Penicilline/Streptomycin, and 1\% L-Glutamine. Injuries to the monolayers were induced using a cylindrical stainless steel indentor with a flat tip (radius $210 \mu \mathrm{m}$ ), 


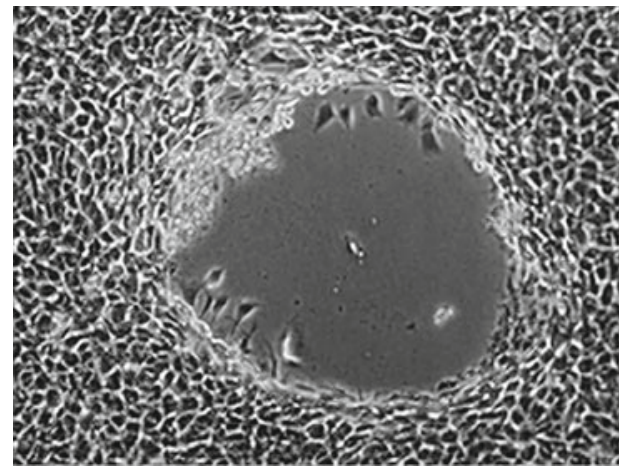

$\min 0$

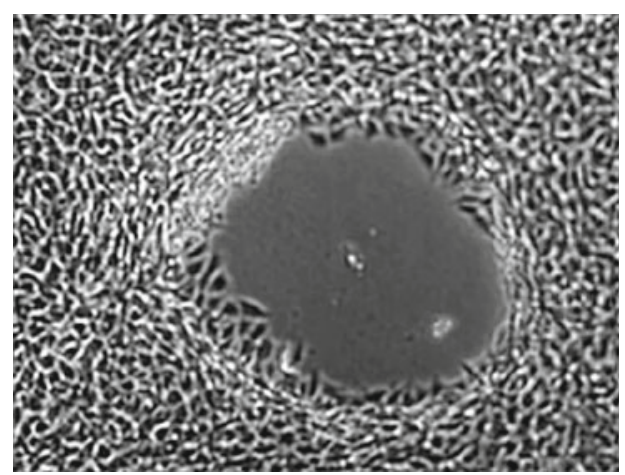

$\min 400$

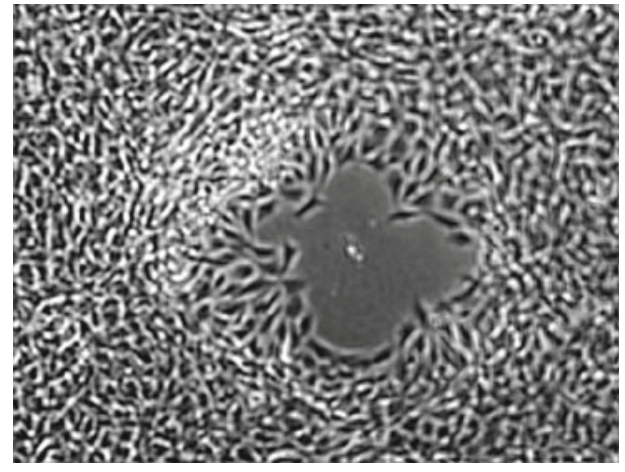

$\min 800$

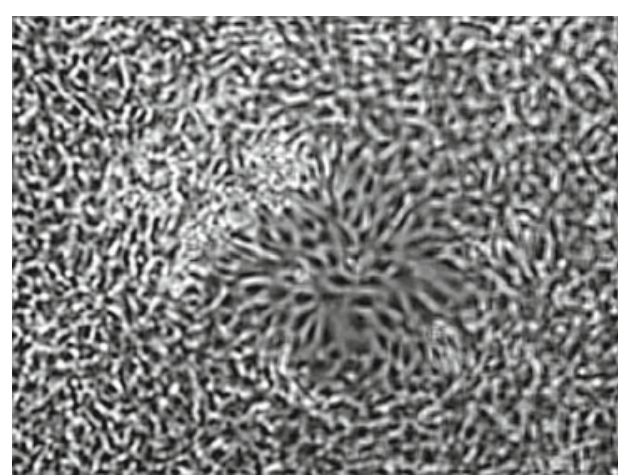

$\min 1200$

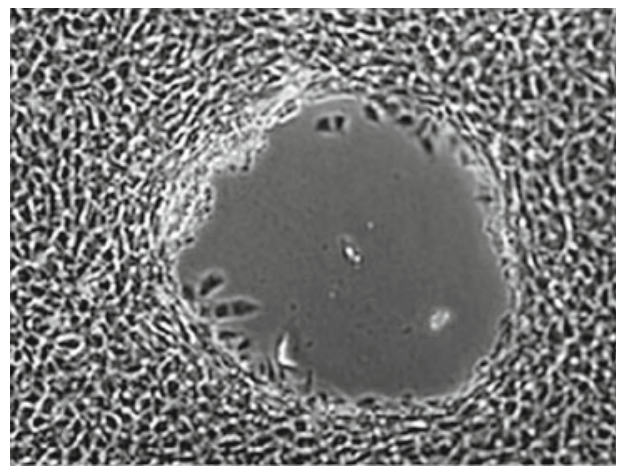

$\min 200$

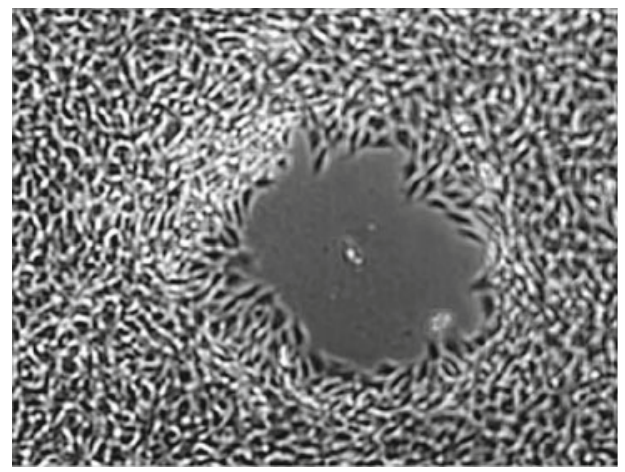

$\min 600$

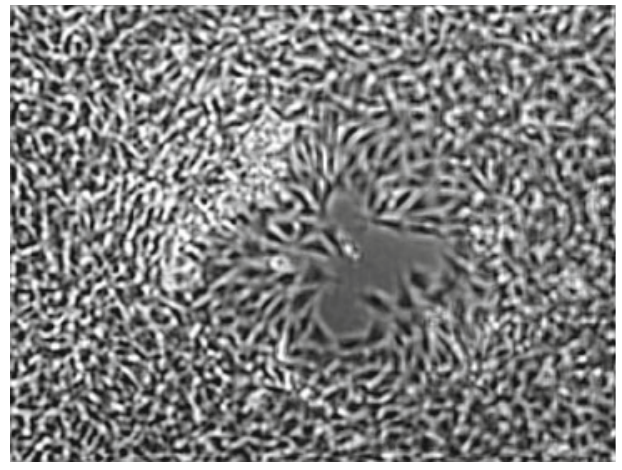

$\min 1000$

Fig. 11 The experimentally observed evolution of the closure of a gap in a colony of fibroblast cells 
which was lowered quasi-statically onto the cultures to cause squash damage to the monolayers using a specially designed apparatus. Injuries were inflicted when the cultures reached 90-100\% confluence. Immediately after delivering the injuries, cultures were placed in an environmental chamber (at a temperature of 37 centigrade), which was also equipped with a phase microscope. Cell migration into the damage sites was then monitored and digitally recorded for $24 \mathrm{~h}$, a period during which cells typically covered the damage site completely. An example of a time course of micrographs of a 3T3 culture following a squash injury is shown in Fig. 11. In Fig. 11, it can be seen that the evolution of the wound gap proceeds similarly to the simulations, for which the results are presented in Figs. 8 and 9. This indicates that, although the model is very preliminary, the simulations predict the right behavior from a qualitative point of view.

\section{Conclusions}

So far, we formulated a model for the simulation of motion of individual cells on a planar substrate. In the model, the motion of the cells is determined by the properties of the substrate, cell elasticity, viability, and randomness. In the model, also processes like cell death and cell division have been taken into account. The results look promising, and a quantitative experimental validation is going to be made. Qualitatively, the model predictions match the experimental observations. Further, the numerical solution coincides well with the analytic solution for two cells. In a future study, we intend to extend the model to deal with cell differentiation such that organ development can be modeled. Furthermore, we intend to compare the current model with partial differential equation models based on the continuum hypothesis.

Open Access This article is distributed under the terms of the Creative Commons Attribution Noncommercial License which permits any noncommercial use, distribution, and reproduction in any medium, provided the original author(s) and source are credited.

\section{References}

Boussinesq J (1885) Application des potentiels ál' étude de l' équilibre et du mouvementdes solides élastiques. Gauthier-Villars, Paris

Burmister D (1945) The general theory of stresses and displacements in layered systems I. J Appl Phys 16:89-94

Burmister D (1945) The general theory of stresses and displacements in layered soil systems II. J Appl Phys 16:126-127

Burmister D (1945) The general theory of stresses and displacements in layered soil systems III. J Appl Phys 16:296-302

Califano JP, Reinhart-King CA (2010) Substrate stiffness and cell area predict cellular traction stresses in single cells and cells in contact. Cell Mol Bioeng 3(1):68-75

Dallon JC, Ehrlich HP (2008) A review of fibroblast populated collagen lattices. Wound Repair Regen 16:472-479

Dallon JC (2010) Multiscale modeling of cellular systems in biology. Curr Opin Coll Interface Sci 15:24-31
Gaffney EA, Pugh K, Maini PK (2002) Investigating a simple model for cutaneous wound healing angiogenesis. J Math Biol 45(4):337374

Gefen A (2010) Effects of virus size and cell stiffness on forces, work and pressures driving membrane invagination in a receptor-mediated endocytosis. J Biomech Eng 132: 084501-1-084501-5 (To appear)

Graner F, Glazier J (1992) Simulation of biological cell sorting using a two-dimensional extended Potts model. Phys Rev Lett 69:20132016

Haga H, Irahara C, Kobayashi R, Nakagaki T, Kawabata K (2005) Collective movement of epithelial cells on a collagen gel substrate. Biophys J 88(3):2250-2256

Hoehme S, Drasdo D (2010) A cell-based simulation software for multi-cellular systems. Bioinformatics 26(20):2641-2642

Javierre E, Vermolen FJ, Vuik C, van der Zwaag S (2009) A mathematical analysis of physiological and morphological aspects of wound closure. J Math Biol 59:605-630

Johnson KL (1985) Contact mechanics. Cambridge University Press, Cambridge

Lemmon CA, Chen CS, Romer LH (2009) Cell traction forces direct fibronectin matrix assembly. Biophys J 96:729-738

Lo CM, Wang HB, Dembo M, Wang YL (2000) Cell movement is guided by the rigidity of the substrate. Biophys J 79(1):144-152

Luding S (2008) Introduction to discrete element methods: basics of contact force models and how to perform the micro-macro transition to continuum theory. Eur J Environ Civil Eng 12(7-8(Special Issue: Alert Course, Aussois)):785-826

Maggelakis SA (2004) Modeling the role of angiogenesis in epidermal wound healing. Discret. Cont. Syst. 4:267-273

Merks MH, Koolwijk P (2009) Modeling morphogenesis in silico and in vitro: towards quantitative, predictive, cell-based modeling. Math Model Nat Phenom 4(4):149-171

Merkel R, Kirchgesner N, Cesa CM, Hoffmann B (2007) Cell force microscopy on elastic layers of finite thickness. Biophys $\mathrm{J}$ 93:3314-3323

Murray JD (2004) Mathematical biology II: spatial models and biomedical applications. Springer, New York

Olsen L, Sherratt JA, Maini PK (1995) A mechanochemical model for adult dermal wound closure and the permanence of the contracted tissue displacement role. J Theor Biol 177:113-128

Plank MJ, Sleeman BD (2004) Lattice and non-lattice models of tumour angiogenesis. Bull Math Biol 66:1785-1819

Reinhart-King CA, Dembo M, Hammer DA (2008) Cell-cell mechanical communication through compliant substrates. Biophys J 95:6044-6051

Sarvestani AS (2010) On the effect of substrate compliance on cellular mobility. J Biochip Tissue Chip 1:101. doi:10.4172/2153-0777. 1000101

Schugart RC, Friedman A, Zhao R, Sen CK (2008) Wound angiogenesis as a function of tissue oxygen tension: a mathematical model. Proc Nat Acad Sci USA 105(7):2628-2633

Schwarz US, Bischofs IB (2005) Physical determinants of cell organization in soft media. Med Eng Phys 27:763-772

Sherratt JA, Murray JD (1991) Mathematical analysis of a basic model for epidermal wound healing. J Math Biol 29:389-404

Vermolen FJ (2009) A simplified finite-element model for tissue regeneration with angiogenesis. ASCE J Eng Mech 135(5):450-461

Vermolen FJ, Javierre E (2009) On the construction of analytic solutions for a diffusion-reaction equation with a discontinuous switch mechanism. J Comput Appl Math 231:983-1003

Wang JHC, Lin J-S (2007) Cell traction force and measurement methods. Biomech Model Mechanobiol 6:361-371

Xue C, Friedman A, Sen CK (2009) A mathematical model of ischemic cutaneous wounds. Proc Nat Acad Sci USA 106(39):16783-16787 